\title{
SQUARE ROOTS OF HYPONORMAL OPERATORS
}

\author{
MEE-KYOUNG KIM \\ Department of Mathematics, Sung Kyun Kwan University, Sowon 440-706, Korea \\ EUNGIL KO \\ Department of Mathematics, Ewha Women's University, Seoul 120-750, Korea
}

(Received 18 February, 1998)

\begin{abstract}
An operator $T \in \mathcal{L}(H)$ is called a square root of a hyponormal operator if $T^{2}$ is hyponormal. In this paper, we prove the following results: Let $S$ and $T$ be square roots of hyponormal operators.

(1) If $\sigma(T) \cap[-\sigma(T)]=\phi$ or $\{0\}$, then $T$ is isoloid (i.e., every isolated point of $\sigma(T)$ is an eigenvalue of $T)$.

(2) If $S$ and $T$ commute, then $S T$ is Weyl if and only if $S$ and $T$ are both Weyl.

(3) If $\sigma(T) \cap[-\sigma(T)]=\phi$ or $\{0\}$, then Weyl's theorem holds for $T$.

(4) If $\sigma(T) \cap[-\sigma(T)]=\phi$, then $T$ is subscalar. As a corollary, we get that $T$ has a nontrivial invariant subspace if $\sigma(T)$ has non-empty interior. (See [3].)
\end{abstract}

1991 Mathematics Subject Classification. 47B20

1. Introduction. Let $H$ and $K$ be separable complex Hilbert spaces, and let $\mathcal{L}(H, K)$ denote the space of all bounded linear operators from $H$ to $K$. If $H=K$, we write $\mathcal{L}(H)$ in place of $\mathcal{L}(H, K)$.

An operator $T$ is called hyponormal if $T^{*} T \geq T T^{*}$, or equivalently if $\|T h\|$ $\geq\left\|T^{*} h\right\|$, for all $h \in H$. We say that an operator $T \in \mathcal{L}(H)$ is a square root of a hyponormal operator if $T^{2}$ is hyponormal. In general, $T^{2}$ can be hyponormal without $T$ being hyponormal. For example, if $T$ is any nilpotent operator of order 2 (i.e., $T^{2}=0$ ), then $T$ is not necessary a hyponormal operator, but is a square root of a hyponormal operator.

A bounded linear operator $S$ on $H$ is called scalar of order $m$ if it possesses a spectral distribution of order $m$; i.e., if there is a continuous unital morphism

$$
\Phi: C_{0}^{m}(C) \rightarrow \mathcal{L}(H)
$$

such that $\Phi(z)=S$, where $z$ stands for the identity function on $\mathbf{C}$ and $C_{0}^{m}(\mathbf{C})$ for the space of compactly supported functions on $\mathbf{C}$, continuously differentiable of order $m$, where $0 \leq m \leq \infty$. An operator is subscalar if it is similar to the restriction of a scalar operator to an invariant subspace.

2. Preliminaries. An operator $T \in \mathcal{L}(H)$ is said to be Fredholm if $\operatorname{ran} T$ is closed and both $\operatorname{ker} T$ and $H / \operatorname{ran} T\left(=\operatorname{ker} T^{*}\right)$ are finite dimensional. The index of a Fredholm operator is defined as 


$$
\text { index } \begin{aligned}
T & =\operatorname{dim} \operatorname{ker} T-\operatorname{dim}(H / \operatorname{ran} T) \\
& =\operatorname{dim} \operatorname{ker} T-\operatorname{dim} \operatorname{ker} T^{*} .
\end{aligned}
$$

The spectrum of $T$ is defined by

$$
\sigma(T)=\{\lambda \in \mathbf{C}: T-\lambda \text { is not invertible }\}
$$

and $\rho(T)=\mathbf{C} \backslash \sigma(T)$ is called the resolvent set of $T$.

The Weyl spectrum of $T$ is defined by

$$
\omega(T)=\{\lambda \in \mathbf{C}: T-\lambda \text { is not Fredholm of index } 0\} .
$$

Clearly, $\omega(T) \subset \sigma(T)$. Furthermore, if $T$ is Fredholm of index 0, we say that $T$ is Weyl.

Let iso $\sigma(T)$ denote the set of isolated points of $\sigma(T), \sigma_{p}(T)$ the set of eigenvalues of $T$, and $\pi_{00}(T)$ the isolated points of $\sigma(T)$ that are eigenvalues of finite multiplicity. If $\omega(T)=\sigma(T)-\pi_{00}(T)$, or equivalently, if $\sigma(T)-\omega(T)=\pi_{00}(T)$, we say that Weyl's theorem holds for $T$. It is known that Weyl's theorem holds for any hyponormal operator. (See [2].)

An operator $T \in \mathcal{L}(H)$ is said to satisfy the single valued extension property if for any open subset $U$ in $\mathbf{C}$, the function

$$
z-T: \mathcal{O}(U, H) \rightarrow \mathcal{O}(U, H)
$$

defined by the obvious pointwise multiplication, is one-to-one, where $\mathcal{O}(U, H)$ denotes the Fréchet space of $H$-valued analytic functions on $U$ with respect to the uniform topology. If, in addition, the function $z-T$ above has closed range on $\mathcal{O}(U, H)$, then $T$ satisfies Bishop's condition $(\beta)$.

Lemma 2.1 [10, Introduction]. Every subscalar operator has property $(\beta)$.

Let $z$ be the coordinate in $\mathbf{C}$ and let $d \mu(z)$, or simply $d \mu$, denote planar Lebesgue measure. Fix a separable, complex Hilbert space $H$ and a bounded (connected) open subset $U$ of $\mathbf{C}$. We shall denote by $L^{2}(u, H)$ the Hilbert space of measurable functions $f: U \rightarrow H$, such that

$$
\|f\|_{2, U}=\left\{\int_{U}\|f(z)\|^{2} d \mu(z)\right\}^{\frac{1}{2}}<\infty .
$$

The space of functions $f \in L^{2}(U, H)$ that are analytic in $U$ (i.e., $\left.\bar{\partial} f=0\right)$ is denoted by

$$
A^{2}(U, H)=L^{2}(U, H) \cap \mathcal{O}(U, H) .
$$

$A^{2}(U, H)$ is called the Bergman space for $U$.

Let us define now a special Sobolev type space. Let $U$ again be a bounded open subset of $\mathbf{C}$ and $m$ a fixed non-negative integer. The vector valued Sobolev space $W^{m}(U, H)$ with respect to $\bar{\partial}$ and of order $m$ will be the space of those functions 
$f \in L^{2}(U, H)$ whose derivatives $\bar{\partial} f, \cdots, \bar{\partial}^{m} f$ in the sense of distributions also belong to $L^{2}(U, H)$. Endowed with the norm

$$
\|f\|_{W^{m}}^{2}=\sum_{i=0}^{m}\left\|\bar{\partial}^{i} f\right\|_{2, U}^{2}
$$

$W^{m}(U, H)$ becomes a Hilbert space contained continuously in $L^{2}(U, H)$.

The linear operator $M$ of multiplication by $z$ on $W^{m}(U, H)$ is continuous and it has a spectral distribution of order $m$, defined by the relation

$$
\Phi_{M}: C_{0}^{m}(\mathbf{C}) \rightarrow \mathcal{L}\left(W^{m}(U, H)\right), \Phi_{M}(f)=M_{f}
$$

Therefore, $M$ is a scalar operator of order $m$.

3. Weyl's theorem. In this section, we show that if $T$ is a square root of a hyponormal operator with the property that $\sigma(T) \cap[-\sigma(T)]=\phi$ or $\{0\}$, then Weyl's theorem holds for $T$.

THEOREM 3.1. If $T$ is a square root of a hyponormal operator with the property that $\sigma(T) \cap[-\sigma(T)]=\phi$ or $\{0\}$, then $T$ is isoloid (i.e., iso $\sigma(T) \subset \sigma_{p}(T)$ ).

Proof. If $\lambda \in$ iso $\sigma(T)$, then $\lambda^{2} \in$ iso $\sigma(T)^{2}$. Since iso $\sigma(T)^{2}=$ iso $\sigma\left(T^{2}\right)$, by the spectral mapping theorem, $\lambda^{2} \in$ iso $\sigma\left(T^{2}\right)$. Since $T^{2}$ is hyponormal, it is isoloid by [11]. Therefore, $\lambda^{2} \in \sigma_{p}\left(T^{2}\right)$. Hence $\lambda^{2} \in \sigma_{p}(T)^{2}$ by [4, Problem 74].

If $\lambda=0$, it is clear that $0 \in \sigma_{p}(T)$.

If $\lambda \neq 0$, then either $\lambda \in \sigma_{p}(T)$ or $-\lambda \in \sigma_{p}(T)$. Since $\lambda \in$ iso $\sigma(T)$ and $\sigma(T) \cap[-\sigma(T)]=\phi$, we know that $-\lambda \notin \sigma(T)$. Therefore $\lambda \in \sigma_{p}(T)$. This completes the proof.

COROLlARY 3.2. If $T$ is a square root of a hyponormal operator with the property that $\sigma(T) \cap[-\sigma(T)]=\phi$ or $\{0\}$, then for any polynomial $p$ we have

$$
\sigma(p(T))-\pi_{00}(p(T))=p\left(\sigma(T)-\pi_{00}(T)\right)
$$

Proof. This is clear from Theorem 3.1 and [9, Proposition 1].

Lemma 3.3. ([5], [12]). Let $S$ and $T$ be commuting operators.

(a) ST is Fredholm if and only if $S$ and $T$ are both Fredholm.

(b) If $S$ and $T$ are both Fredholm, then index $S T=\operatorname{index} S+\operatorname{index} T$.

THEOREM 3.4. If $S$ and $T$ are commuting square roots of hyponormal operators, then $S T$ is Weyl if and only if $S$ and $T$ are both Weyl.

Proof. If $S T$ is Weyl, then $S T$ is Fredholm of index 0. By Lemma 3.3, we know that $(S T)^{2}$ is Fredholm and index $(S T)^{2}=$ index $S T+$ index $S T=0$. Since 
$(S T)^{2}=S^{2} T^{2}$ is Fredholm, $S^{2}$ and $T^{2}$ are both Fredholm, by Lemma 3.3. Since $S^{2}$ and $T^{2}$ are hyponormal, index $S^{2} \leq 0$ and index $T^{2} \leq 0$. Since index $(S T)^{2}=0$, Lemma 3.3 implies that

$$
\begin{aligned}
0 & =\text { index }(S T)^{2} \\
& =\text { index } S^{2}+\text { index } T^{2} .
\end{aligned}
$$

Therefore, index $S^{2}=0=$ index $T^{2}$.

Since $S^{2}$ and $T^{2}$ are Fredholm, it follows from Lemma 3.3 that $S$ and $T$ are Fredholm and

$$
0=\operatorname{index} S^{2}=\text { index } S+\text { index } S \text {. }
$$

Therefore, index $S=0$. Similarly, index $T=0$. We conclude that $S$ and $T$ are both Weyl.

Conversely, if $S$ and $T$ are both Weyl, then $S$ and $T$ are both Fredholm of index 0. By Lemma 3.3, $S T$ is Fredholm and

$$
\text { index } S T=\operatorname{index} S+\operatorname{index} T=0 \text {. }
$$

Therefore, $S T$ is Weyl.

LEMMA 3.5. Let $T$ be a square root of a hyponormal operator with the property that $\sigma(T) \cap[-\sigma(T)]=\phi$ or $\{0\}$. If $\lambda \in \pi_{00}(T)$, then $\lambda^{2} \in \pi_{00}\left(T^{2}\right)$.

Proof. By Corollary 3.2 with $p(t)=t^{2}$, we have

$$
\sigma\left(T^{2}\right)-\pi_{00}\left(T^{2}\right)=\left(\sigma(T)-\pi_{00}(T)\right)^{2} .
$$

Let $\lambda \in \pi_{00}(T)$. If $\lambda^{2} \notin \pi_{00}\left(T^{2}\right)$, then $\lambda^{2} \in \sigma\left(T^{2}\right)-\pi_{00}\left(T^{2}\right)$. It follows that $\lambda^{2} \in(\sigma(T)$ $\left.-\pi_{00}(T)\right)^{2}$.

If $\lambda=0$, it is clear that $0 \in \sigma(T)-\pi_{00}(T)$ and so we have a contradiction.

If $\lambda \neq 0$, then either $\lambda \in \sigma(T)-\pi_{00}(T)$ or $-\lambda \in \sigma(T)-\pi_{00}(T)$. Since $\lambda \in \pi_{00}(T)$ and $\sigma(T) \cap[-\sigma(T)]=\phi,-\lambda \notin \sigma(T)$. Therefore, $\lambda \in \sigma(T)-\pi_{00}(T)$ and we have a contradiction. Thus $\lambda^{2} \in \pi_{00}\left(T^{2}\right)$.

THEOREM 3.6. If $T$ is a square root of a hyponormal operator with the property that $\sigma(T) \cap[-\sigma(T)]=\phi$ or $\{0\}$, then Weyl's theorem holds for $T$.

Proof. It suffices to show that $\lambda \in \sigma(T)-\pi_{00}(T)$ if and only if $\lambda \in \omega(T)$.

If $\lambda \in \sigma(T)-\pi_{00}(T)$, then $\lambda^{2} \in\left(\sigma(T)-\pi_{00}(T)\right)^{2}$. By Corollary 3.2, we have

$$
\lambda^{2} \in \sigma\left(T^{2}\right)-\pi_{00}\left(T^{2}\right) .
$$

Since $T^{2}$ is hyponormal, a theorem of Coburn [2] implies that

$$
\lambda^{2} \in \sigma\left(T^{2}\right)-\pi_{00}\left(T^{2}\right)=\omega\left(T^{2}\right) .
$$


Since $\omega\left(T^{2}\right) \subset \omega(T)^{2}$ by $[\mathbf{1}], \lambda^{2} \in(T)^{2}$.

If $\lambda=0$, it is clear that $0 \in \omega(T)$.

If $\lambda \neq 0$, then either $\lambda \in \omega(T)$ or $-\lambda \in \omega(T)$. Since $\lambda \in \sigma(T), \omega(T) \subset \sigma(T)$, and $\sigma(T) \cap[-\sigma(T)]=\phi,-\lambda \notin \omega(T)$. Therefore, $\lambda \in \omega(T)$.

Conversely, let $\lambda \in \omega(T)$.

Claim. If $\lambda \notin \sigma(T)-\pi_{00}(T)$, then $\lambda^{2} \notin\left(\sigma(T)-\pi_{00}(T)\right)^{2}$.

We verify the claim above. If $\lambda=0$, it is clear. Let $\lambda \neq 0$. If $\lambda^{2} \in\left(\sigma(T)-\pi_{00}(T)\right)^{2}$, then either $\lambda \in \sigma(T)-\pi_{00}(T)$ or $-\lambda \in \sigma(T)-\pi_{00}(T)$. Since $\lambda \notin \sigma(T)-\pi_{00}(T),-\lambda \in \sigma(T)-\pi_{00}(T)$. But $-\lambda \notin \sigma(T)$, since $\lambda \in \omega(T) \subset \sigma(T)$ and $\sigma(T) \cap[-\sigma(T)]=\phi$. Hence we have a contradiction.

Let us come back now to the proof of Theorem 3.6. If $\lambda \notin \sigma(T)-\pi_{00}(T)$, then $\lambda^{2} \notin\left(\sigma(T)-\pi_{00}(T)\right)^{2}$ by the Claim. Now Corollary 3.2 implies that

$$
\lambda^{2} \notin \sigma\left(T^{2}\right)-\pi_{00}\left(T^{2}\right) .
$$

Since $T^{2}$ is hyponormal, a theorem of Coburn [2] implies that $\lambda^{2} \notin \sigma\left(T^{2}\right)-\pi_{00}\left(T^{2}\right)=\omega\left(T^{2}\right)$. Therefore, $\lambda^{2} \in \sigma\left(T^{2}\right)-\omega\left(T^{2}\right)$. Thus $T^{2}-\lambda^{2}$ is Weyl.

If $\lambda=0$, then $T^{2}$ is Weyl. Hence $T$ is Weyl by Theorem 3.4. Thus $0 \notin \omega(T)$, and so we have a contradiction.

Let $\lambda \neq 0$. By Lemma 3.3, $T+\lambda$ and $T-\lambda$ are both Fredholm. Since $\lambda \in \omega(T) \subset \sigma(T)$ and $\sigma(T) \cap[-\sigma(T)]=\phi,-\lambda \notin \sigma(T)$. Therefore, $T+\lambda$ is invertible and so $T+\lambda$ is Weyl. By Lemma 3.3, we have

$$
0=\operatorname{index}\left(T^{2}-\lambda^{2}\right)=\operatorname{index}(T+\lambda)+\operatorname{index}(T-\lambda) .
$$

Therefore, index $(T-\lambda)=0$. Hence $T-\lambda$ is Weyl. Thus $\lambda \notin \omega(T)$, and so we have a contradiction. Thus $\lambda \in \sigma(T)-\pi_{00}(T)$.

COROLLARY 3.7. If $T$ is a square root of a hyponormal operator with the property that $\sigma(T) \cap[-\sigma(T)]=\phi$ or $\{0\}$ and $N$ is a nilpotent operator commuting with $T$, then Weyl's theorem holds for $T+N$.

Proof. It follows from Theorem 3.6 and [9, Theorem 3].

4. Subscalarity. In this section, we show that if $T$ is a square root of a hyponormal operator with the property that $\sigma(T) \cap[-\sigma(T)]=\phi$ then $T$ is subscalar.

Lemma 4.1. ([10], Proposition 2.1). For every bounded disk $D$ in $\mathbf{C}$ there is a constant $C_{D}$, such that for an arbitrary operator $T \in \mathcal{L}(H)$ and $f \in W^{2}(D, H)$ we have

$$
\|(I-P) f\|_{2, D} \leq C_{D}\left(\left\|(T-z)^{*} \bar{\partial} f\right\|_{2, D}+\left\|(T-z)^{*} \bar{\partial}^{2} f\right\|_{2, D}\right),
$$

where $P$ denotes the orthogonal projection of $L^{2}(D, H)$ onto the Bergman space $A^{2}(D, H)$. 
Lemma 4.2. Let $T$ be a square root of a hyponormal operator with the property that $\sigma(T) \cap[-\sigma(T)]=\phi$ and let $D$ be a bounded disk which contains $\sigma(T)$. Then the operator $V: H \rightarrow H(D)$, defined by

$$
V h=1 \otimes h+\overline{(z-T) W^{2}(D, H)} \quad(=1 \tilde{\otimes} h),
$$

is one-to-one and has closed range, where $H(D)=W^{2}(D, H) / \overline{(z-T) W^{2}(D, H)}$ and $1 \otimes h$ denotes the constant function sending any $z \in D$ to $h$.

Proof. Let $h_{i} \in H$ and $f_{i} \in W^{2}(D, H)$ be sequences such that

$$
\lim _{i \rightarrow \infty}\left\|(z-T) f_{i}+1 \otimes h_{i}\right\|_{w^{2}}=0
$$

Then, by the definition of the norm of a Sobolev space, (1) implies that

$$
\lim _{i \rightarrow \infty}\left\|(z-T) \bar{\partial}^{j} f_{i}\right\|_{2, D}=0
$$

for $j=1,2$. From (2), we get

$$
\lim _{i \rightarrow \infty}\left\|\left(z^{2}-T^{2}\right) \bar{\partial}^{j} f_{i}\right\|_{2, D}=0
$$

for $j=1,2$. Since $T^{2}$ is hyponormal,

$$
\lim _{i \rightarrow \infty}\left\|\left(\bar{z}^{2}-T^{* 2}\right) \bar{\partial}^{j} f_{i}\right\|_{2, D}=0 .
$$

Since $z-T$ is invertible for $z \in D \backslash \sigma(T)$, the equation (2) implies that

$$
\lim _{i \rightarrow \infty}\left\|\bar{\partial}^{j} f_{i}\right\|_{2, D \backslash \sigma(T)}=0 .
$$

Therefore,

$$
\lim _{i \rightarrow \infty}\left\|\left(\bar{z}-T^{*}\right) \bar{\partial}^{j} f_{i}\right\|_{2, D \backslash \sigma(T)}=0 .
$$

Since $\sigma(T) \cap[-\sigma(T)]=\phi$ and $\sigma(T)^{*}=\sigma\left(T^{*}\right)$, it is clear that $T^{*}+\bar{z}$ is invertible for $z \in \sigma(T)$. Therefore, from the equation (3) we have

$$
\lim _{i \rightarrow \infty}\left\|\left(\bar{z}-T^{*}\right) \bar{\partial}^{j} f_{i}\right\|_{2, \sigma(T)}=0 .
$$

Hence, from (4) and (5) we obtain

$$
\lim _{i \rightarrow \infty}\left\|\left(\bar{z}-T^{*}\right) \bar{\partial}^{j} f_{i}\right\|_{2, D}=0 .
$$


Then, by Lemma 4.1, we have

$$
\lim _{i \rightarrow \infty}\left\|(I-P) f_{i}\right\|_{2, D}=0
$$

where $P$ denotes the orthogonal projection of $L^{2}(D, H)$ onto $A^{2}(D, H)$. By (1) and (7), we have

$$
\lim _{i \rightarrow \infty}\left\|(z-T) P f_{i}+1 \otimes h_{i}\right\|_{2, D}=0
$$

Let $\Gamma$ be a circle in $D$ such that $\sigma(T)$ lies inside $\Gamma$. Assume that $\Gamma$ is described once counterclockwise. Then

$$
\lim _{i \rightarrow \infty}\left\|P f_{i}(z)+(z-T)^{-1}\left(1 \otimes h_{i}\right)\right\|=0
$$

uniformly for $z$ in $\Gamma$. Hence, by the Riesz functional calculus,

$$
\lim _{i \rightarrow \infty}\left\|\frac{1}{2 \pi i} \int_{\Gamma} P f_{i}(z) d z+h_{i}\right\|=0 .
$$

But $\int_{\Gamma} P f_{i}(z) d z=0$. Hence, $\lim _{i \rightarrow \infty} h_{i}=0$.

THEOREM 4.3. If $T$ is a square root of a hyponormal operator with the property that $\sigma(T) \cap[-\sigma(T)]=\phi$, then $T$ is subscalar of order 2 .

Proof. Consider an arbitrary bounded open disk $D$ in $\mathbf{C}$ that contains $\sigma(T)$ and the quotient space

$$
H(D)=W^{2}(D, H) / \overline{(z-T) W^{2}(D, H)}
$$

endowed with the Hilbert space norm. Let $M\left(=M_{z}\right)$ be the multiplication operator by $z$ on $W^{2}(D, H)$. Then $M$ is a scalar operator of order 2 and its spectral distribution is

$$
\Phi: C_{0}^{2}(\mathbf{C}) \rightarrow \mathcal{L}\left(W^{2}(D, H)\right), \quad \Phi(f)=M_{f}
$$

where $M_{f}$ is the operator of multiplication by $f$. Since $M$ commutes with $z-T, \tilde{M}$ on $H(D)$ is still a scalar operator of order 2 , with $\tilde{\Phi}$ as a spectral distribution.

Let $V$ be the operator

$$
V h=1 \otimes h\left(=1 \otimes h+\overline{(z-T) W^{2}(D, H)}\right),
$$

from $H$ into $H(D)$, denoting by $1 \otimes h$ the constant function $h$. Then $V T=\tilde{M} V$. Since $V$ is one-to-one and has closed range by Lemma 4.2, $T$ is subscalar of order 2. 
COROLlaRY 4.4. Let $T$ be a square root of a hyponormal operator with the property that $\sigma(T) \cap[-\sigma(T)]=\phi$. If $\sigma(T)$ has interior in the plane, then Thas a nontrivial invariant subspace.

Proof. It follows from Theorem 4.3 and [3].

COROLlARY 4.5. If $T$ is a square root of a hyponormal operator with the property that $\sigma(T) \cap[-\sigma(T)]=\phi$, then $T$ has the property $(\beta)$.

Proof. It follows from Theorem 4.3 and Lemma 2.1.

COROLlaRY 4.6. Let $T$ be a square root of a hyponormal operator with the property that $\sigma(T) \cap[-\sigma(T)]=\phi$. If $A$ is any quasiaffine transform of $T$ (i.e., there exists a one-to-one $X$ with dense range such that $X A=T X)$, then $\sigma(T) \subset \sigma(A)$.

Proof. It is clear from Corollary 4.5 and [8].

\section{REFERENCES}

1. S. K. Berberian, The Weyl spectrum of an operator, Indiana Univ. Math. J. 20 (1970), $529-544$

2. L. A. Coburn, Weyl's theorem for nonnormal operators, Michigan Math. J. 13 (1966), 285-288.

3. J. Eschmeier and B. Prunaru, Invariant subspaces for operators with Bishop's property $(\beta)$ and thick spectrum, J. Funct. Anal. 94 (1990), 196-222.

4. P. R. Halmos, A Hilbert space problem book (Springer-Verlag, 1982).

5. R. Harte, Invertibility and singularity for bounded linear operators (Marcel Dekker, New York, 1988).

6. E. Ko, Algebraic and triangular n-hyponormal operators, Proc. Amer. Math. Soc. 123 (1995), 3473-3481.

7. E. Ko, k-Quasihyponormal operators are subscalar, Integral Equations Operator Theory 28 (1997), 492-499.

8. E. Ko, On a Clary theorem, Bull Korean Math. Soc. 33 (1996), 29-33.

9. K. Oberai, On the Weyl spectrum II, Illinois J. Math. 21 (1977), 84-90. 395.

10. M. Putinar, Hyponormal operators are subscalar, J. Operator Theory 12 (1984), 385-

11. J. G. Stampfli, Hyponormal operators, Pacific J. Math. 12 (1962), 1453-1458.

12. A. E. Taylor and D. C. Lay, Introduction to functional analysis (John Wiley and Sons, New York, 1980). 